\title{
L'AUTRE RIVE: LES IRANIENS AUX ÉMIRATS ARABES UNIS, ENTRE VISIBILITÉ ET INVISIBILITÉ
}

Amin MogHADAM *

Si les échanges entre les rives du golfe Persique se poursuivent au sein d'un territoire réticulaire dans le Sud et dans le Nord, par le biais du commerce légal ou illégal, l'importance de la présence des Iraniens aux Émirats arabes unis témoigne de nouvelles tendances migratoires qui font écho à un processus plus large de globalisation et de métropolisation. Plus de 500000 Iraniens vivent et travaillent aux Émirats arabes unis dont la population avoisine les 4 millions d'habitants. Il n'est plus nécessaire de rappeler que le développement urbain de Doubaï est dû en partie à la présence de migrants venus des quatre continents. Mais si la majorité de la population de Doubaï est originaire d'Asie du Sud et qu'un habitant sur deux est né en Inde, la dimension iranienne, ancienne, reste prégnante et $80 \%$ des Iraniens des Émirats se trouvent à Doubaï.

Comme pour chaque terre d'immigration, les migrants sont répartis et canalisés dans différents secteurs d'activité. La diversité de la présence iranienne rend difficile l'identification de la totalité des secteurs d'activité où celle-ci intervient, d'autant plus que cette configuration hétéroclite semble empêcher la constitution de nouvelles formes communautaires, surtout entre l'ancienne génération et la nouvelle, issue d'un mouvement migratoire récent. Cette hétérogénéité résulte de l'origine diverse des mouvements migratoires iraniens vers les Émirats, dont chacun comporte sa propre économie morale et politique. Néanmoins, la tendance générale va vers une qualification

* Amin Moghadam est urbaniste-sociologue à l'université Paris VII. Il est diplômé de l'École nationale des ponts et chaussées (ENPC) et doctorant au laboratoire GREMMO, université Lyon II. L'article a été rédigé dans le cadre de l'axe Golfe du programme CITADAIN (comparer l'intégration des territoires et l'adaptation du droit dans le monde arabe et indien) financé par l'ANR. 
croissante des profils migratoires, de manière à répondre aux exigences d'une société en quête d'hypermodernité.

Nous voudrions montrer ici le rôle du mouvement migratoire iranien dans le développement et le processus de métropolisation de Doubaï. Or, aujourd'hui, la présence iranienne aux Émirats arabes unis prend des formes multiples, entre visibilité et invisibilité, entre un communautarisme régional et des initiatives individuelles depuis les grands centres urbains de l'Iran. On remarque, sur plus d'un siècle, le passage d'une forme migratoire ancienne fondée sur des réseaux et valeurs communautaires, à une forme récente marquée par l'individuation. Cela correspond aux évolutions politiques et sociétales des sociétés d'origine et d'accueil, à savoir l'Iran et les Émirats arabes unis. Néanmoins, ce passage à une nouvelle forme de migration ne se fait pas au détriment de la première forme de migration : la volonté des Émirats d'atteindre une société basée sur la connaissance pose la question de savoir jusqu'à quand cette économie communautaire et périphérique pourra subsister.

\section{UNE INVERSION DU MOUVEMENT MIGRATOIRE LIÉE AU CONTEXTE GÉOPOLITIQUE}

Les pérégrinations entre les deux côtes, arabe et persane, ont marqué la vie des villes concernées depuis des siècles. Il s'agit d'une migration à l'échelle régionale, où le golfe Persique prend toute sa fonction d'interface, au sens géographique du terme, pour répondre aux besoins d'une économie locale des villages des deux côtes. Cette présence s'explique souvent par les affinités confessionnelles et langagières des habitants du Sud de l'Iran et ceux des pays méridionaux du Golfe et par les événements historiques qui ont marqué les pays respectifs de chaque côte et qui ont accentué ou ralenti les migrations dans un sens ou dans un autre.

Le développement du port de Siraf ${ }^{1}$ en Iran au IX ${ }^{\mathrm{e}}$ siècle et l'élargissement de l'échelle des échanges commerciaux dans l'océan Indien, depuis les villes lointaines de l'Extrême-Orient jusqu'à celles des côtes du golfe Persique, avaient rendu très attractives Siraf et les villes environnantes pour les marchands. La répercussion de ces dynamiques sur l'environnement proche comme à Bastak et à Galeh Dar et jusqu'à au port de Bouchehr à plus de $200 \mathrm{~km}$ est à l'origine de l'immigration d'un nombre important d'Arabes. Si l'on en croit la tradition à Bastak ou à Lar et d'après quelques rares écrits sur l'histoire de ces rivages, les premiers immigrants étaient des Arabes venus des villages de la côte sud du Golfe qui se sont installés dans les villages de Bastak, de Lar et au port de Lengeh où les terres étaient fertiles et le commerce florissant. Majoritairement appelées des Hûwalah ${ }^{2}$ et réparties dans la région, ces tribus se caractérisaient par les voyages qu'elles effectuaient entre les deux

1. S. M.N. Priestman, The Rise of Siraf: Long-Term Development of Trade Emporia within the Persian Gulf, International Congress of Siraf Port, novembre 2005.

2. A. Montigny, "Les Arabes de l'autre rive », Cahiers d'études sur la Méditerranée orientale et le monde turco-iranien, n² 22, juillet-décembre 1996, pp. 51-81. 
rives, arabe et persane. Ces tribus, arabes et sunnites ${ }^{3}$, ont créé des alliances avec les habitants de Bastak, de Lar et de Bandar Lengeh et ont même pris, de temps à autre, le contrôle de ces derniers. Avec la constitution d'un Etatnation fort au $\mathrm{XV}^{\mathrm{e}}$ siècle sous les Safavides autour des identités persane et chiite, les Arabes sunnites installés depuis plusieurs siècles dans le Sud de l'Iran ont été pourchassés par les gouverneurs locaux.

Quelques siècles plus tard, une première vague d'immigration massive d'Iraniens vers les côtes arabes annonce un inversement de la tendance migratoire. En 1910, une importante communauté marchande, souvent arabophone et sunnite, quitta les côtes iraniennes pour les côtes méridionales en raison de la mise en place en 1902 de barrières douanières par des représentants belges mandatés en Iran pour apporter des solutions de protection à l'économie iranienne. Cette loi, qui introduisait des droits de douane très élevés sur toutes les marchandises importées et exportées dans les ports iraniens, a tourné au profit du développement de Doubaï qui a ainsi remplacé progressivement Bandar Lengeh, port dominant jusqu'alors. Doubaï est alors devenu «non seulement un centre de distribution pour le commerce avec intérieur, l'oasis de Buraimi en particulier, mais il reçut aussi des marchandises en provenance de l'Inde, par voilier ou par vapeur (un service régulier hebdomadaire fonctionna à partir de juin 1904), marchandises qui étaient réexportées vers la Perse et d'autres pays de la région ${ }^{4}$ », écrit Frauke Heard-Bey. D'après elle, la population qui a migré du littoral iranien vers le port de Doubaii avait gardé des liens avec la côte perse et faisait en sorte que les marchandises arrivent en Perse en contournant les postes douaniers. Des décennies plus tard, Doubaï - dont on peut, d'une certaine manière, comparer la situation actuelle dans le golfe Persique face à la République islamique d'Iran, à ce que fut celle de Hongkong, dans le delta de la rivière des Perles, à proximité de la Chine communiste - a permis à l'Iran de contourner l'embargo américain.

De nos jours, la migration massive des Iraniens vers les Émirats est, en grande partie, la conséquence du contexte géopolitique et des relations entre les pays arabes du Golfe et l'Iran. Après la révolution et durant les années de la guerre Iran-Irak (1980-1989) et même après, alors que l'Iran se consacrait à une économie de guerre puis à la reconstruction, son voisin émirien s'est largement développé et la ville de Doubaï s'est vue qualifiée de " cité-État ${ }^{5}$ " ou promue au rang de " cité globale ${ }^{6}$ ". Elle est devenue un paradis de la

3. Sans qu'il s'agisse réellement d'une donnée historique vérifiable, les habitants de Bastak et de Lar semblent insister sur une alliance avec la famille al-Nahyan, la famille régnante de l'émirat d'Abou Dhabi!

4. F. Heard-Bey, Les Émirats arabes unis, Paris, Karthala, 1999.

5. Roland Marchal (dir.), Doubaï, cité globale, Paris, CNRS, coll. " Espaces et Milieux », 2001.

6. G. Bastien, A. Maraut, B. Telle, Et après le pétrole? Risques et enjeux géopolitiquesfinanciers pour les Émirats arabes unis, Paris, Harmattan, coll. « Entreprises et management », 2005. 
consommation et des transactions financières. Doubaï a pu profiter de ses avantages comparatifs par rapport à la côte iranienne: des infrastructures développées, l'efficacité de son aéroport, des installations portuaires, des facilités de crédits, de paiement et de change ainsi que la présence de banques étrangères. Chaque année, un nombre élevé d'investisseurs iraniens quittent leur pays pour Doubaï avec d'importants capitaux (ces départs ont été accentués après l'élection du président Ahmadinejad ${ }^{7}$ ). Fariba Adelkhah se demande si Doubaï, devenu le centre de réexportation vers l'Iran des produits notamment américains depuis l'embargo, n'est pas devenu aussi « la véritable capitale économique de la République islamique ${ }^{8}$ ».

Bien que l'augmentation des échanges commerciaux et l'intensification des relations d'interdépendance aient fortifié les relations entre les deux pays, la méfiance marque toujours les relations bilatérales entre l'Iran et les pays arabes du Golfe. Après la révolution, les dirigeants politiques des pays arabes du golfe Persique ont été considérés par l'imam Khomeyni comme " idolâtres » et traîtres à l'Islam en raison de leur alliance avec les États-Unis. Le conflit entre les Émirats arabes unis et l'Iran pour le contrôle de trois îles du golfe Persique a resurgi régulièrement dans les débats depuis plus de trente ans. La tension entre les deux protagonistes se répercute sur la présence iranienne aux Émirats. Cela se traduit à la fois dans le discours des autorités locales des EAU par une minimisation de la contribution des Iraniens dans le développement du pays et chez le migrant par une démarche individuelle de dissimulation de sa propre identité iranienne. Nous avons pu vérifier ces deux hypothèses à l'occasion des enquêtes et des entretiens réalisés lors de deux missions ${ }^{9}$ aux Émirats arabes unis en 2007 et en 2008, succédant à un travail de recherche sur la côte iranienne et dans les îles iraniennes du Golfe mené en 2006.

\section{L'EMPREINTE IRANIENNE SUR LE DOUbAÏ D'HIER VISIBLE DANS LE DoubAÏ D'AUJOURD'HUI}

Malgré le visage futuriste des gratte-ciel et des autoroutes de Doubaï, le présent rappelle le passé. La crique de Doubaï, Al-Khor, qui sépare le quartier de Deira à l'est et celui de BurDoubaï à l'ouest, abrite toujours de nombreux boutres (dhow dans le langage des pays arabes du Golfe et lenj en Iran) qui battent pavillon émirien, mais dont les matelots et les marins parlent le persan, le jazirati (dialecte des îles iraniennes du golfe Persique) ou le pachtou. Les principaux acteurs de l'économie du Golfe d'hier sont aujourd'hui relégués à une économie périphérique et

7. Voir T. Coville, «L’Économie iranienne, rupture ou continuité? », Géoéconomie, $\mathrm{n}^{\circ}$ 36, hiver 2005-2006, pp. 97-107.

8. F. Adelkhah, "Doubaï, capitale économique de l’Iran? », dans R. Marchal (dir.), Doubaï, cité globale, Paris, CNRS, coll. «Espaces et Milieux », 2001, pp. 40-41.

9. Notre réflexion s'inscrit dans l'axe Golfe du programme CITADAIN (comparer l'intégration des territoires et l'adaptation du droit dans le monde arabe et indien) financé par l'ANR. 
participent au commerce régional par le « bas $10 »$ : ils assurent le transport de toute une gamme de marchandises détaxées à Doubaï et prisées pour la modicité de leur prix à leur arrivée à Qeshm, Bandar Abbas et Kish en Iran. La crique de Doubaï est toujours le lieu d'accueil d'acteurs de l'économie de l'ombre, de boutres et de petites barques noctambules qui sillonnent les abords du détroit d'Ormuz, relayés en Iran par des conducteurs de $4 \mathrm{X} 4$ sur des routes reculées ou encore des « femmes parachutistes ${ }^{11}$ ». Il s'agit d'un trafic mal connu et inchiffrable qui se noue dans les réseaux anciens de familles, d'amitiés et de relations. Cela recouvre aussi le passage de clandestins iraniens ou afghans ayant transité par l'Iran dont l'existence est connue, mais l'ampleur difficile à préciser, qui fournit une main-d'œuvre de journaliers peu qualifiés pour des emplois ${ }^{12}$. Les commerçants à Doubaï, souvent considérés comme des contrebandiers en Iran, traversent les frontières, souvent illégitimes à leurs yeux, pour assurer la survie des " territoires circulatoires ${ }^{13}$ du golfe Persique qui peinent à se maintenir face aux évolutions de Doubaï et à son orientation rapide vers une économie de la connaissance ${ }^{14}$.

Si dans le quartier de Deira, situé dans le vieux Doubaï et au bord de la crique, les enseignes iraniennes sont plus nombreuses que dans d'autres quartiers de la ville, elles ne sont pas les seules marques de la présence iranienne. À la concentration de marchands iraniens qui témoigne d'une présence ancienne qui continue à exister au profit du trafic des boutres à proximité s'ajoute l'activité des hôtels remplis d'Iraniens qui vont à l'assaut des souks et des centres commerciaux de Doubaï ou passent une nuit en transit avant d'atteindre Abou Dhabi afin de se rendre à l'ambassade des États-Unis dans l'espoir d'y obtenir leur visa. La visibilité de la présence iranienne dans ce quartier n'est plus représentative de son poids économique réel. À l'ouest de la crique, le quartier du patrimoine de Bastakia, enserré aujourd'hui dans le quartier de BurDoubaï, témoigne de l'ancienneté de la présence de populations venues du Sud de l'Iran à Doubaï. Condamné à être démoli en 1988 dans le plan de modernisation de Doubaï, mais préservé par une correction de ce dernier l'année suivante, on y retrouve aujourd'hui les maisons rénovées et caractérisées par des

10. A.Tarrius, La mondialisation par le bas. Les nouveaux nomades de l'économie souterraine, Paris, Balland, 2002.

11. Il s'agit des femmes qui servent à transporter des produits de luxe depuis les zones franches iraniennes vers l'intérieur du pays. "Femmes parachutistes » est leur appellation dans le langage courant sur les zones franches. Voir A. Moghadam, $L a$ zone franche: implications économiques et territoriales du projet de "Qeshm Free Area » dans le Sud iranien, mémoire de master d'aménagement et urbanisme, université Paris IV-Sorbonne, 2006.

12. P. Cadène, B. Dumortier, "L'impact politique des flux migratoires dans les pays du Conseil de coopération du Golfe », L'espace politique, n 4, 2008, http://www. espacepolitique.org/documents/pdf/EP4_Migrations_golfe.pdf

13. A. Tarrius, op. cit.

14. P. Cadène, «Société de la connaissance et développement aux Émirats arabes unis », Maghreb Machrek, n 195, printemps 2008. 
tours à vent les Barjeel (Bâd Gir en persan) que l'on trouve aussi en Iran de la ville de Yazd jusqu'aux abords du golfe Persique. À l'image des habitants, ce style architectural, traversant le Golfe, rappelle la continuité culturelle entre les côtes méridionale et septentrionale. Ce style est aujourd'hui au service de certaines nouvelles constructions luxueuses de Doubaï telle que Madinat Al jumeira où les labyrinthes de couloirs et de balcons éclairés par une lumière artificiellement tamisée sont censés rappeler les histoires mystérieuses des Mille et une nuits. Ce n'est peut-être pas un hasard si Doubaï cherche aujourd'hui son histoire et son identité dans ces quartiers. Si aucune des salles successives du musée de Doubaï, relatant les phases de la success story doubaïote, n'évoque l'influence de la présence iranienne sur le développement de la ville, quelques mètres plus loin, au cœur des ruelles nouvellement réhabilitées du "Quartier de patrimoine », contrastant avec les médinas hollywoodiennes néoorientalistes qui surgissent du désert, la présence iranienne devient indéniable. Ce quartier est aujourd'hui le lieu d'expression d'un très grand nombre d'artistes iraniens à travers des événements culturels comme la Creek Art Fair et grâce à la présence d'un nombre important de galeries d'origine iranienne comme la XVA galerie qui expose les œuvres d'artistes iraniens les plus prisés comme les photos d'Abbas Kiarostami ou celles de Bahman Jalali, mondialement connus et reconnus dans les milieux artistiques.

Cette implantation, bien que localisée en partie dans les quartiers dits « anciens » de Doubaï, s'inscrit dans les nouvelles ambitions de la métropole émergente ${ }^{15}$. Si Bastakia hébergeait autrefois marchands et marins des ports et des villages du Sud de l'Iran, il est aujourd'hui le lieu d'expression de l'intelligentsia iranienne venue des quatre coins du monde, à commencer par Fereydoun Ave, copropriétaire de la XVA galerie, dont les œuvres d'art ont circulé dans le monde entier, et qui détient un rôle influent dans le commerce d'œuvres d'art entre Paris, Téhéran et Doubaï. C'est notamment la volonté de globalité qui incite les autorités de la ville à dessiner une nouvelle image, non pas uniquement par des gratte-ciel et des voies expresses, mais aussi par la création de lieux culturels dont Bastakia, recomposé au service de l'histoire et de l'art contemporain, n'est pas le seul exemple. Dans un urbanisme décomplexé ${ }^{16}$, cette volonté se lit dans les nouvelles constructions des centres commerciaux tels que le fameux Madinat al-Jumeirah, cité plus haut, ou, plus ambitieux encore dans le même registre, le " prestigieux " complexe de Wafi city. Ici pas de style architectural revendiqué, simplement la glorification des emblèmes d'un passé hétéroclite étranger à la région à travers la reconstitution des Pyramides ou du Parthénon. Les Iraniens ne sont pas en reste lorsqu'il s'agit de se faire une place dans ce nouveau créneau des artefacts recréés d'une tradition qui se réinvente: un restaurant au nom inspiré de l'Iran antique, Persia Persia, occupe une place stratégique dans le complexe, et diffuse de la musique persane à longueur de journée, tandis que

15. Ibid.

16. Ibid. 
le personnel, aux vêtements persans traditionnels et à l'accent californien ${ }^{17}$, est anglophone.

\section{L'IMPORTANCE VOILÉE OU DÉVOILÉE D'UNE NOUVELLE GÉNÉRATION D'IRANIENS}

Dans la course vers une " société de la connaissance » telle que la définit Philippe Cadène, caractérisée par l'importance accordée aux travailleurs qualifiés, la présence iranienne évolue pour s'adapter aux exigences de cette " conurbation littorale transfrontalière ${ }^{18}$ » qui cherche à mieux s'intégrer dans les dynamiques mondiales. Entre visibilité et invisibilité, les « nouveaux Iraniens » des EAU participent depuis plus d'une décennie à un large éventail d'activités et s'adaptent aux évolutions des secteurs économiques prédominants. Les statistiques fournies par la Chambre de commerce et d'industrie des Émirats prennent en compte les activités qui sont officiellement tenues par les Iraniens de nationalité iranienne. Or, les Iraniens aux Émirats ne sont pas toujours des Iraniens au sens de l'administration, ce pour deux raisons. La première immigration iranienne dans les principautés arabes au début du siècle a souvent cherché à s'arabiser (ou à se réarabiser), y compris en adoptant des patronymes persans, pour mieux s'intégrer dans la société d'accueil, et ce par le biais d'affinités ethniques, religieuses et linguistiques. Au moment de l'indépendance, les membres de cette communauté implantée bien avant la découverte du pétrole remplissaient les conditions requises pour accéder au statut envié de "national » au même titre que les tribus arabes locales. Cette assimilation va jusqu'à la négation de son ascendance iranienne comme en témoigne, par exemple, le refus de parler le persan, qui a fait l'objet d'une transmission familiale, en public, ou de fréquenter d'autres Iraniens. Hussein Sajwani, le président du troisième groupe multinational de promotion immobilière des Émirats arabes unis qui opère dans plus de 18 pays, semble ainsi ne perdre aucune occasion de mettre en avant sa nationalité émirienne même si son appartenance iranienne n'échappe ni aux Iraniens de Doubaï qui observent avec une certaine béatitude sa réussite, ni aux autorités locales des Émirats. Néanmoins, la stratégie d'Hussein Sajwani de dissimulation de son identité persane et chiite ne l'a pas empêché d'apporter l'aide la plus importante du secteur privé aux sinistrés du tremblement de terre de Bam en Iran.

Un autre groupe d'Iraniens est constitué de ceux qui ont vécu longtemps en Europe ou aux États-Unis, souvent depuis le début de la révolution iranienne, et qui ont pu obtenir la nationalité du pays d'adoption, mais ont gardé des liens avec leur pays d'origine. Les opportunités offertes dans les villes des Émirats les ont incités à développer des affaires à Doubaï ou à Ras al-Khaïmah, à proximité de leur terre natale et dans un

17. Les États-Unis, en particulier la Californie du Sud, ont accueilli une large fraction de la diaspora iranienne qui a dû faire face à beaucoup de suspicions après le 11 septembre et dont certains membres sont repartis dans le Golfe, mais sur la rive arabe.

18. B. Dumortier, « Doubaï », Images Économiques du Monde, SEDES, 2006. 
environnement économique plus stable et plus libéral qu'en Iran. Le cas de Nasser Hashempour, le vice-président de l'Iranian Business Council, en est l'illustration. Enfant, il a quitté l'Iran en 1967, a vécu au Canada et aux États-Unis avant de décider d'émigrer à Doubaï en 1988. Il est en lien direct avec l'Iran par le biais d'une compagnie qui importe des tapis et des fruits secs ainsi que des produits pétrochimiques. Ces Iraniens qui ont vécu à l'ouest, bien que ne figurant pas dans les statistiques officielles en tant que ressortissants iraniens, sont désireux de tisser des liens avec la jeune génération venue des grandes villes iraniennes depuis la fin des années 1980 et qui, quant à elle, est à la recherche d'une meilleure qualité de vie qu'en Iran, où la situation actuelle ne leur semble pas très prometteuse. Â cela s'ajoute la catégorie particulière des étudiants, de l'ordre de 11500 dans l'ensemble des Émirats arabes unis, très présents à Doubaï, où ils suivent majoritairement des cursus en anglais dans des filières appliquées.

Malgré l'insuffisance des statistiques de la Chambre de commerce et d'industrie de Doubaï concernant la présence iranienne, quelques chiffres donnent une idée de son importance. En 2006, sur environ 500000 Iraniens habitant aux Émirats, 400000 sont installés à Doubaï (l'agglomération dans son ensemble compte environ 2 millions d'habitants) et $20 \%$ des investissements enregistrés dans l'ensemble des centres commerciaux de Doubaï ont été effectués par des Iraniens. À la même date, 7000 sociétés ont été enregistrées sous la rubrique " nationalité iranienne » à la Chambre de commerce et d'industrie de Doubaï. Néanmoins seulement 3000 à 4000 de ces sociétés auraient une activité, les autres étant considérées comme " fictives » par le chargé d'affaires économiques du consulat d'Iran à Doubaï. Certaines sociétés s'enregistrent uniquement pour profiter des avantages fiscaux de Doubaï et l'utilisent comme plateforme pour l'exportation de produits iraniens vers d'autres continents. En ce qui concerne les échanges entre les deux pays, la valeur des échanges officiels non pétroliers s'élève à 11,7 milliards de dollars en 2007 dont 9,2 milliards d'exportations depuis les Émirats vers l'Iran. En outre, selon notre interlocuteur au consulat d'Iran à Doubaï, $40 \%$ des importations d'essence de l'Iran se font depuis les Émirats, ce qui n'est pas compris dans les chiffres fournis, qui concernent les échanges non pétroliers. Les exportations et surtout les réexportations les plus importantes pour Doubaï - et également pour Abou Dhabi sont à destination de l'Iran alors que ce dernier ne figure pas parmi les premiers fournisseurs des EAU ( $27^{\mathrm{e}}$ rang). Ce taux de réexportation important résulte, d'une part, de l'embargo américain interdisant l'exportation des produits américains vers l'Iran et, d'autre part, du manque de compétitivité des installations portuaires en Iran.

De même, le taux d'investissement iranien aux Émirats a augmenté de $50 \%$ entre 2005 et 2006 avec une croissance notamment dans le secteur immobilier passant de 200 milliards de dollars à 300 milliards de dollars. En ce qui concerne ce dernier secteur, un des plus importants aujourd'hui 
dans l'économie de Doubaï ${ }^{19}$, les Iraniens détiendraient entre $10 \%$ et $30 \%$ des transactions immobilières avec une augmentation de $10 \%$ entre 2006 et 2007. Ces investissements suivent la logique d'un processus de métropolisation littorale aux allures de mégalopolisation qui s'étendrait sur toute la côte depuis Ras al-Khaïmah au nord jusqu'à Abou Dhabi au sud en passant par la conurbation d'Ajman-Chardjah-Doubaï. Ainsi $31 \%$ des villas luxueuses du complexe d'al-Hamra à Ras al-Khaïmah auraient été achetées par des Iraniens. Les projets de la société de courtage immobilier Oriental Real Estate sont à cet égard éloquents. Touraj Bakhtiar, fondateur et manager de la société, se vante de ses origines iraniennes. Il a déclaré dans un entretien accordé à la presse locale et la chaîne CNBC: "Je suis à $100 \%$ iranien et le sang oriental coule dans toutes mes veines », cela même s'il a vécu depuis l'âge de cinq ans en Grande-Bretagne et en France. Sa société contribue aujourd'hui à la commercialisation de projets immobiliers depuis Doubaï jusqu'à Ajman et Ras al-Khaïmah. Promulguée en 2006, une loi qui permet aux étrangers d'être propriétaire sans restriction de temps ou d'être usufruitiers pendant quatre-vingt-dixneuf ans ${ }^{20}$ a accéléré le développement de cette agence créée la même année. Celle-ci coopère notamment dans le cadre du projet de Sport City avec les grands promoteurs immobiliers tels que Falak properties mais opère aussi dans les "zones de libre tenure " nouvellement mises en place par la loi citée ci-dessus ${ }^{21}$. Une grande partie des employés de la société sont des Iraniens. Avec des bureaux installés dans les Malls et Shopping centers de Doubaï, les agents commerciaux d'Oriental Real Estate savent attirer la clientèle iranienne fortunée à la recherche de nouveaux investissements aux Émirats et, si nécessaire, parlent persan pour faciliter la communication.

Alors que, dans certains cas, les origines iraniennes sont assumées, revendiquées et même médiatisées, ailleurs elles sont davantage « voilées ». L'émirat de Ras al-Khaïmah à l'extrémité nord des Émirats profite aujourd'hui, d'un point de vue économique, de sa proximité avec Doubaï avec une voie express qui les relie en une heure. Bien que les autorités locales dénient les profits tirés de la proximité avec Doubaï, cette dernière est incontestablement une des raisons principales de la concentration des sociétés et des services dans cet émirat de 250000 habitants. Intégré à son tour dans le processus de métropolisation littorale, Ras al-Khaïmah tente

19. B. Dumortier, « De l'appropriation coutumière aux lois foncières: adaptation du droit et expansion urbaine aux Émirats arabes unis ", Études foncières, n 132 , mars-avril 2008.

20. Ibid.

21. Au passage, il est important de rappeler que l'émergence de ce type d'acteur dans le secteur d'immobilier et sa concomitance avec les lois facilitant l'accès des étrangers à la propriété contribue à un " urbanisme ségrégatif ", ce que Brigitte Dumortier explique dans l'article cité précédemment. Il en ira sans doute de même pour l'immigration iranienne dont les origines deviendront à l'avenir choisies voire ségrégatives! 
aujourd'hui de jouer sur sa position géographique en tendant les bras aussi bien aux autres émirats de la Fédération qu'aux les pays voisins. Saqr port, le port principal de la ville et le plus proche du vis-à-vis iranien, le port de Bandar Abbas, a traditionnellement gardé des liens avec la côte iranienne et les îles du golfe Persique pour le transport des produits agricoles et des passagers. Deux ferries de 40 passagers partent tous les jours depuis Ras al-Khaïmah à destination de Kish. Deux autres ports sont en construction et permettront d'intensifier les transports avec le reste du pays et les pays voisins. De même, la compagnie aérienne Ras Airways envisage de mettre en place des vols entre Ras al-Khaïmah et les villes iraniennes de Chiraz, Ispahan et Téhéran. Du côté iranien, Kish Airlines, de la zone franche insulaire de Kish en Iran, assure deux vols hebdomadaires entre l'île et Ras al-Khaïmah.

D'après les entretiens effectués, une grande partie des entreprises enregistrées sont sans activité productive réelle et constituent surtout une domiciliation. Les frais que chaque société paie au gouvernement local de Ras al-Khaïmah pour s'enregistrer ou renouveler sa licence d'activité lui garantissent sa pérennité officielle dans l'émirat sans pour autant qu'elle soit obligée d'y avoir son siège principal et lui permettent l'accès aux autres émirats. Les Iraniens, contraints par la législation de leur pays et par les sanctions internationales, sont les premiers à user de ces facilités. C'est le cas d'une société de fabrication de rideaux, d'origine iranienne, basée à Ras al-Khaïmah dont les réunions de travail se déroulent dans les grands hôtels de Doubaï, son principal marché. La plupart des sociétés iraniennes utilisent aussi la plate-forme de Ras al-Khaïmah pour les activités bancaires, le commerce et la réexportation des produits vers l'Iran.

Certains Iraniens de retour d'Occident, particulièrement d'Europe, ont toutefois réussi à inscrire leur présence dans la production locale: les actions d'une société importante, RAK Petropak, dont les activités tournent autour $\mathrm{du}$ " packaging » et de la production de plastique, en lien avec l'entreprise de cimenterie la plus importante du pays, RAKCC, sont détenues à $50 \%$ par des Iraniens et $50 \%$ par le gouvernement local. Néanmoins, les autorités locales tendent à minimiser, voire à dissimuler, la participation iranienne aux projets de Ras al-Khaïmah même si elles n'hésitent pas à s'appuyer sur des Iraniens pour tisser davantage de liens avec les entrepreneurs de l'autre rive.

\section{CONCLUSION: UN RÊVE DOUX-AMER}

Dans Café Setareh, un film du réalisateur iranien Saman Moghadam sorti en 2006, Saloumeh, une jeune Iranienne, suite aux promesses de son prétendant, Elbi, rêve de s'installer avec ce dernier sur une des îles artificielles de Doubaï pour échapper à la pauvreté et à la tristesse de sa condition. Elle dessine alors son île avec la mosquée de son quartier délabré du Sud de Téhéran et une grande maison luxueuse où sa grand-mère handicapée, son futur mari et elle-même vivront dans la paix et la prospérité. Les vains efforts d'Elbi pour émigrer aux Émirats orientent celui-ci vers des activités 
illégales et il finit par se retrouver en prison. Saloumeh, les larmes aux yeux, accroche le dessin onirique de son île de Doubaï au-dessus de son lit et continue à attendre son bien-aimé.

L'imaginaire de cette jeune fille, beaucoup de jeunes Iraniens le partagent. Néanmoins, ce désir de Doubaï laisse un goût amer sur le palais des Iraniens. Cette amertume naît du développement spectaculaire des Émirats qui laisse l'Iran loin derrière. Les discours populaires, mais aussi étatiques, reflètent le ressentiment des Iraniens face au développement de la rive méridionale du Golfe qui résulte en partie de la présence historique d'une communauté iranienne très dynamique. Ces sentiments ou ressentiments affectent également les représentations des acteurs politiques. La situation frontalière des zones franches iraniennes telles que les îles de Qeshm et de Kish, proches de la limite des eaux iraniennes, est l'occasion d'évaluer les politiques publiques qui y sont mises en place. Le « retard » de ces zones est mis en relation avec la concurrence des zones franches émiriennes qui attirent des entreprises iraniennes au lieu d'être expliqué par les caractéristiques du modèle politico-économique de l'Iran dont la conception et la gestion des zones franches iraniennes se ressentent. De même, Téhéran a pendant longtemps partagé les mêmes ambitions que Doubaï. Le projet d'aéroport international de Téhéran dans les années 1970 et la compagnie Iranair avaient vocation à devenir ce que sont aujourd'hui l'aéroport de Doubaï et la compagnie Emirates.

Le succès de Doubaï, aujourd'hui insérée dans des réseaux métropolitains de villes globales, renvoie aux politiques publiques iraniennes d'autant plus qu'une série d'acteurs iraniens de niveau social divers qui pratiquent des déplacements réguliers entre les deux pays relatent l'ascension rapide de Doubaï. Ces éléments contribuent à la fabrication d'une image, certes idyllique, de Doubaï en Iran, mais en attendant que l'Iran sorte de son impasse politico-économique actuelle, Saloumeh, la jeune fille démunie de Saman Moghadam, continuera à nourrir des rêves de prospérité à Doubaï! 